\title{
Les convictions créationnistes et/ou évolutionnistes d'enseignants de biologie : une étude comparative dans dix-neuf pays
}

\author{
Pierre Clément ${ }^{1}$, Marie-Pierre Quessada ${ }^{2}$ \\ 1 Didactique de la biologie, LEPS-LIRDHIST, Université Lyon 1, 38 Bd Niels Bohr, 69622 Villeurbanne cedex, France \\ 2 Didactique de la biologie, LIRDEF, Université Montpellier 2 et IUFM, 2 place Marcel Godechot, BP 4152, \\ 34092 Montpellier cedex 5, France
}

Les liens entre la naissance ainsi que le développement de la science moderne et les trois grandes religions monothéistes - juive, chrétienne et musulmane - ont été l'objet de plusieurs études : la conception d'un dieu créateur et ordonnateur d'un univers soumis à des lois physiques a historiquement ouvert la possibilité d'étudier ces lois, de les mettre en évidence et de les respecter ${ }^{1}$. Les conflits entre science et religion n'en ont pas moins été nombreux, conduisant progressivement à l'acceptation par ces trois religions monothéistes de l'autonomie de la pensée scientifique, donc des grands faits et théories scientifiques validés par la communauté scientifique. Ainsi, le pape Jean-Paul II a reconnu en 1996 que "de nouvelles connaissances conduisent à ne plus considérer la théorie de l'évolution comme une simple hypothèse ». Les évolutionnistes actuels comprennent aussi bien des agnostiques ou des athées que des croyants (chrétiens, juifs, musulmans) qui ne s'opposent en rien à l'enseignement de l'évolution ni aux recherches

Auteur correspondant : P. Clément,

Pierre.Clement@univ-lyon1.fr

Voir dans les numéros précédents (NSS, 15, 3 et 4 ; NSS, 16, 1) d'autres contributions au dossier Évolution et créationnisme.

${ }^{1}$ Les références bibliographiques seraient nombreuses à citer. Je me limiterai au dossier récent «Dieu contre Darwin » de la revue L'Histoire ( ${ }^{\circ} 328$, février 2008), avec en particulier les articles de J.-P. Filu, «Au nom de Mahomet » (46-48), de J.-C. Attias, «Du côté des rabbins » (49-51) et de J.-R. Armogathe, « Revenons à une lecture symbolique de la Bible» (52-57). scientifiques dans ce domaine, auxquelles ils peuvent même contribuer.

Tout autre est la position des courants fondamentalistes qui connaissent depuis peu un regain important au sein même de ces grandes religions. La plupart des écrits récents sur ce regain analysent les procès successifs qui ont eu lieu aux États-Unis (Lecourt, 1998; Arnould, 2007; Picq, 2007) et le poids croissant des mouvements évangélistes - les «born-again Christians » (George, 2007). Ils discutent aussi les arguments créationnistes fondés sur une lecture fondamentaliste de la Bible. L'ossature de l'argumentation créationniste (Clément, 2002) est d'opposer «l'harmonie de la nature » au " hasard aveugle», passant sous silence que les processus évolutifs combinent hasard et sélection naturelle ${ }^{2}$, pour obliger le lecteur à reconnaître que le hasard seul ne peut tout expliquer et devrait être remplacé par une construction intelligente orchestrée par un grand Horloger ou, plus récemment, par un plan interne (Intelligent Design).

Face à ce regain du créationnisme, les scientifiques qui ont contre-attaqué ont surtout présenté les faits

\footnotetext{
${ }^{2}$ Quand ils évoquent la sélection naturelle, les créationnistes le font sans lien avec les processus évolutifs : ils diabolisent cette notion en la réduisant au "darwinisme social», rendant ainsi les théories de l'évolution responsables du stalinisme autant que du nazisme : voir L'Atlas de la création (Harun Yahya) diffusé à certains enseignants et universitaires début 2007 en France, puis plus largement encore aux États-Unis.
} 
de l'évolution ${ }^{3}$. L'ampleur de l'impact des thèses créationnistes reste jusqu'ici mal évaluée. La «petite géographie du créationnisme» de P. Picq (op. cit., p. 33-56) accorde une large place aux États-Unis, puis à l'Australie et au Canada. En dehors de la France, la situation n'y est rapidement évoquée (en quelques lignes) que pour quelques pays européens (p. 43-46) et pour deux autres pays (Kenya et Turquie, p. 47-49). Notons, par ailleurs, qu'aucun de ces travaux n'a concerné jusqu'ici l'analyse des conceptions $\mathrm{d}^{\prime}$ enseignants du primaire ou du secondaire ${ }^{4}$.

C'est dire l'importance de la recherche menée depuis septembre 2004 dans le cadre du projet Biohead-Citizen ${ }^{5}$ pour analyser les conceptions d'enseignants du primaire et du secondaire dans 19 pays : 7044 enseignants interrogés dans 13 pays européens, 5 pays africains et au Liban (Moyen-Orient) (Tableau). Ce projet de recherche comporte deux volets (Carvalho et Clément, 2007; Clément et Carvalho, 2007) :

- d'une part, l'analyse des programmes et manuels scolaires dans chacun des 19 pays, ainsi que leur comparaison. En ce qui concerne l'évolution (un des six thèmes de ce projet), nos premiers résultats ont été récemment publiés (Quessada et al., à paraître; Clément et al., 2007). Ils sont complétés, pour la France, par une analyse historique des programmes et manuels scolaires aux XIXe et XXe siècles (Quessada et Clément, 2007a et b ; Quessada et Clément, 2006). Les résultats de ces analyses, passionnants, ne peuvent pas être résumés ici faute de place.

- d'autre part l'analyse des conceptions d'enseignants et de futurs enseignants. N'est présentée ici qu'une petite partie non encore publiée de ces résultats. La plupart sont en cours d'analyse ${ }^{6}$.

Dans chaque pays, six échantillons ont été soumis à un long questionnaire (144 questions, questionnaire rédigé collectivement, puis soumis à un test pilote et à différentes procédures de validation ${ }^{7}$ ) : des enseignants et de futurs enseignants du primaire, du secondaire pour les matières biologie et langue principale / lettres. Trente à cinquante enseignants minimum ont été interrogés pour chacun de

\footnotetext{
${ }^{3}$ Le document «Science, Evolution, and Creationism » est intéressant à consulter à cet égard, car il est signé par un grand nombre de scientifiques connus (National Academy of Science, 2008). Il peut être téléchargé gratuitement sur le site suivant : http://www.nap.edu/catalog/11876.html

4 Citons cependant, dans les recherches relevant de la didactique de la biologie, Aroua (2006) et Zineddine (2003).

5 «Biology, Health and Environmental Education for better Citizenship » : STREP CIT2-CT-2004-506015, E.C., Brussels, FP6, coordonné par G. Carvalho (Portugal), P. Clément (France) et F. Bogner (Allemagne), 2004-2008.

6 Nos premiers résultats publiés sur le thème «Évolution » sont Quessada et al. (2007) et Munoz et al. (2007).

7 Ces aspects méthodologiques sont résumés dans Clément et Carvalho (2007).
}

Tableau. Effectif des enseignants et futurs enseignants interrogés dans les 19 pays.

\begin{tabular}{cccc}
\hline $\begin{array}{c}\text { Pays } \\
\text { (sigle) }\end{array}$ & Pays & $\begin{array}{c}\text { Total } \\
\text { échan- } \\
\text { tillons }\end{array}$ & $\begin{array}{c}\text { dont des } \\
\text { biologistes }\end{array}$ \\
\hline BF & Burkina Faso & 296 & 92 \\
CY & Chypre & 322 & 63 \\
DE & Allemagne & 365 & 109 \\
DZ & Algérie & 217 & 57 \\
EE & Estonie & 182 & 60 \\
FI & Finlande & 306 & 121 \\
FR & France & 732 & 249 \\
GB & Grande-Bretagne & 154 & 106 \\
HU & Hongrie & 334 & 108 \\
IT & Italie & 559 & 119 \\
LB & Liban & 722 & 212 \\
LT & Lituanie & 316 & 98 \\
MA & Maroc & 330 & 128 \\
MT & Malte & 198 & 41 \\
PL & Pologne & 311 & 100 \\
PT & Portugal & 350 & 102 \\
RO & Roumanie & 273 & 93 \\
SN & Sénégal & 324 & 114 \\
TN & Tunisie & 753 & 318 \\
Total & & $\mathbf{7 0 4 4}$ & $\mathbf{2 ~ 2 9 0}$ \\
\hline
\end{tabular}

ces six échantillons dans les 19 pays. Dans certains (Tunisie, Liban, France, Italie), ce nombre a été plus important pour tester des hypothèses complémentaires. Au total, 7044 enseignants ou futurs enseignants ont été interrogés, dont un tiers environ de biologistes (Tableau).

Quinze questions portaient sur l'évolution : six sur le créationnisme vs l'évolutionnisme; deux sur le finalisme; une sur la séparation hommes/animaux; six sur des connaissances relatives aux processus de l'évolution. Nous présentons surtout, ci-dessous, les résultats relatifs à une des questions sur le créationnisme vs l'évolutionnisme. Les résultats relatifs aux autres questions sur ce thème précis sont très voisins. Nous terminerons en évoquant les premières analyses déjà réalisées et publiées à partir des quinze questions sur l'évolution.

Le libellé de la question, dont les résultats sont présentés sur les figures 1 et 2, est le suivant:

«A64. Avec laquelle de ces quatre propositions êtesvous le plus en accord? (Cochez seulement une réponse.)

$\square$ Il est certain que l'origine de la vie est le résultat de phénomènes naturels.

$\square$ L'origine de la vie peut être expliquée par des phénomènes naturels sans avoir besoin de l'hypothèse que Dieu a créé la vie.

$\square$ L'origine de la vie peut être expliquée par des phénomènes naturels qui sont sous le contrôle de Dieu.

$\square$ Il est certain que Dieu a créé la vie. » 


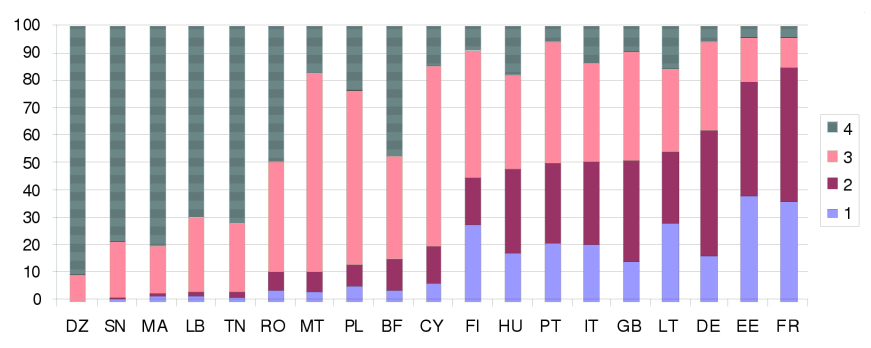

Fig. 1. Réponses des 2290 enseignants biologistes (en service ou en formation) à la question A64 : pourcentage des 4 items par pays (voir Tab. pour les sigles des pays).

P. Clément avait testé, amélioré puis validé cette question au préalable sur des étudiants et des enseignants tunisiens, lors des cours de DEA de didactique de la biologie qu'il donne chaque année depuis 1998 à l'ISEFC de Tunis. Son objectif est triple :

- différencier les réponses évolutionnistes (les deux premiers items) des réponses créationnistes ;

- au sein de ces dernières, différencier les créationnistes radicaux (le $4^{\mathrm{e}}$ item) de ceux qui concilient une création divine initiale avec des processus évolutifs contrôlés par Dieu mais non niés (les «créationnistesévolutionnistes »;

- au sein des évolutionnistes, enfin, distinguer une position carrée, presque dogmatique, d'une position plus nuancée, tout aussi évolutionniste mais mentionnant Dieu sans le faire intervenir.

Les résultats représentés par les figures 1 et 2 sont éloquents.

A) Avec 1 à $2 \%$ de créationnistes radicaux, la France fait figure d'exception (elle est, avec l'Estonie, l'un des deux seuls pays où environ la moitié des enseignants interrogés est agnostique ou athée). Dans sept autres pays européens (Allemagne, Finlande, Grande-Bretagne, Portugal, Italie Lituanie et Hongrie), le pourcentage de créationnistes radicaux reste relativement faible (<20\%).

B) À l'opposé, les créationnistes radicaux sont extrêmement majoritaires (70 à $90 \%$ ) dans cinq pays non européens, qui sont musulmans (Tunisie, Sénégal, Maroc et Algérie) ou aux deux tiers musulmans (Liban).

C) Entre ces deux pôles, se situent cinq pays : deux où les créationnistes radicaux représentent environ la moitié des enseignants interrogés (Burkina Faso et Roumanie) et trois où ils représentent moins de la moitié, surtout chez les biologistes (Chypre, Malte et Pologne).

La différence entre les conceptions des (futurs) enseignants biologistes et non biologistes est significative dans onze pays (Allemagne, Burkina, Chypre, Finlande, Hongrie, Italie, Liban, Maroc, Pologne, Portugal, Tunisie). Dans certains d'entre eux (Chypre, Malte, Pologne), elle porte surtout sur le nombre de «créationnistesévolutionnistes »(item 3), qui, chez les biologistes,

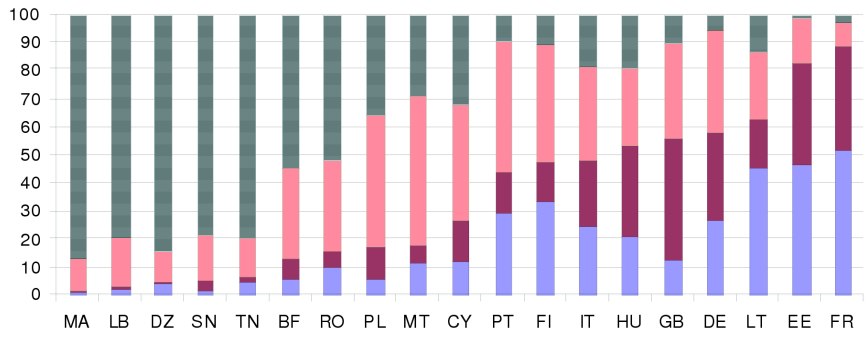

Fig. 2. Réponses des 4792 enseignants non biologistes (en service ou en formation) à la question A64 : pourcentage des 4 items par pays (voir Tab. pour les sigles des pays).

remplacent les créationnistes radicaux (item 4), ce qui est une façon pour eux de conjuguer une croyance en Dieu, très répandue dans ces pays, avec l'acceptation de l'évolutionnisme.

Bien d'autres comparaisons et commentaires seraient possibles à partir des résultats obtenus pour cette seule question : par exemple, en ce qui concerne le groupe C, défini ci-dessus, il est possible de remarquer qu'il comprend les deux seuls pays à majorité orthodoxe dans notre choix de pays (Chypre et Roumanie), ainsi que deux pays très catholiques pratiquants (Pologne et Malte); enfin, les enseignants interrogés au Burkina Faso sont aux deux tiers chrétiens, ce qui peut expliquer la légère différence avec le Sénégal où presque tous les enseignants sont musulmans. A contrario, le tiers de chrétiens libanais (pour deux tiers de musulmans) ne différencie pas le Liban des quatre pays très majoritairement musulmans.

Les réponses aux autres questions relatives aux opinions créationnistes vs évolutionnistes convergent tout à fait avec les résultats qui viennent d'être exposés pour la question A64. Les réponses aux autres questions sur l'évolution ne sont pas toutes corrélées à celles-ci. Ainsi, celles sur les connaissances relatives aux processus évolutifs suivent une autre logique de distribution, avec parfois des interactions avec les valeurs : par exemple, le « dessein intelligent » est assez lié aux réponses créationnistes, tandis que le rôle du hasard ainsi que celui de la sélection naturelle sont liés à l'évolutionnisme. Toutes ces cohérences sont faciles à lire dans les graphes obtenus par des analyses multivariées. Nous n'avons la place d'en présenter qu'une ici (Fig. 3), mais d'autres sont consultables dans nos premières publications (Quessada et al., 2007; Munoz et al., 2007).

Les 19 pays se répartissent, comme pour la question A64 (Fig. 1 et 2), entre un pôle créationniste (à gauche de l'axe horizontal de la figure 3) et un pôle évolutionniste (à droite de cet axe). Des résultats non illustrés par la figure 3 montrent une corrélation très forte entre les convictions créationnistes et le degré de croyance religieuse. La corrélation est encore plus forte avec le degré de pratique religieuse. Les taux les plus importants de créationnistes 
(a)

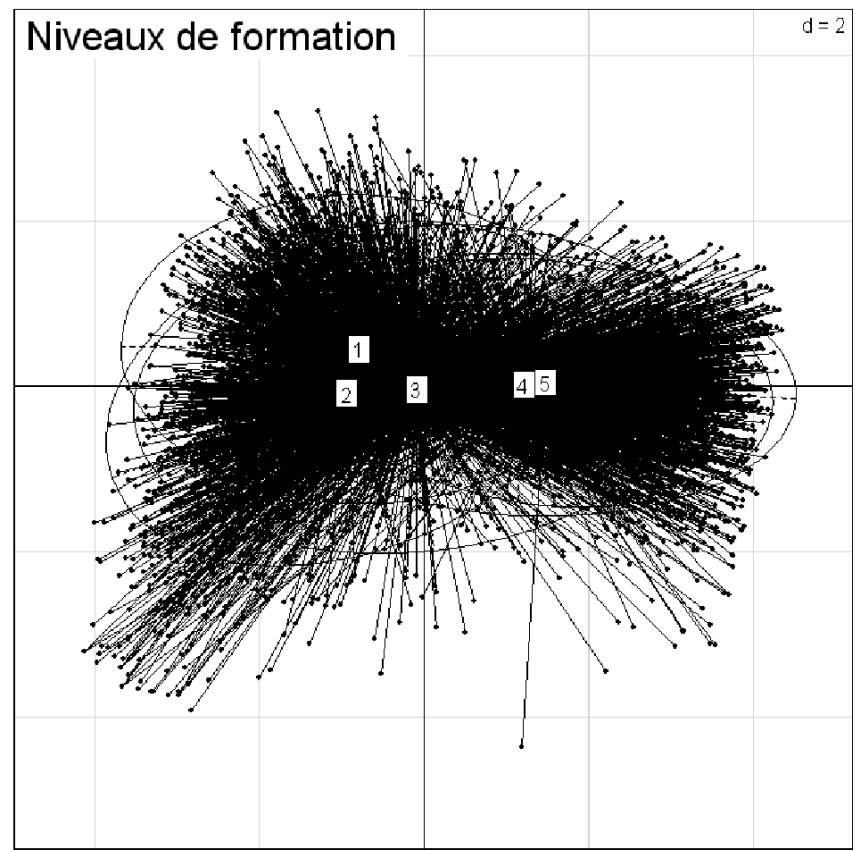

(b)

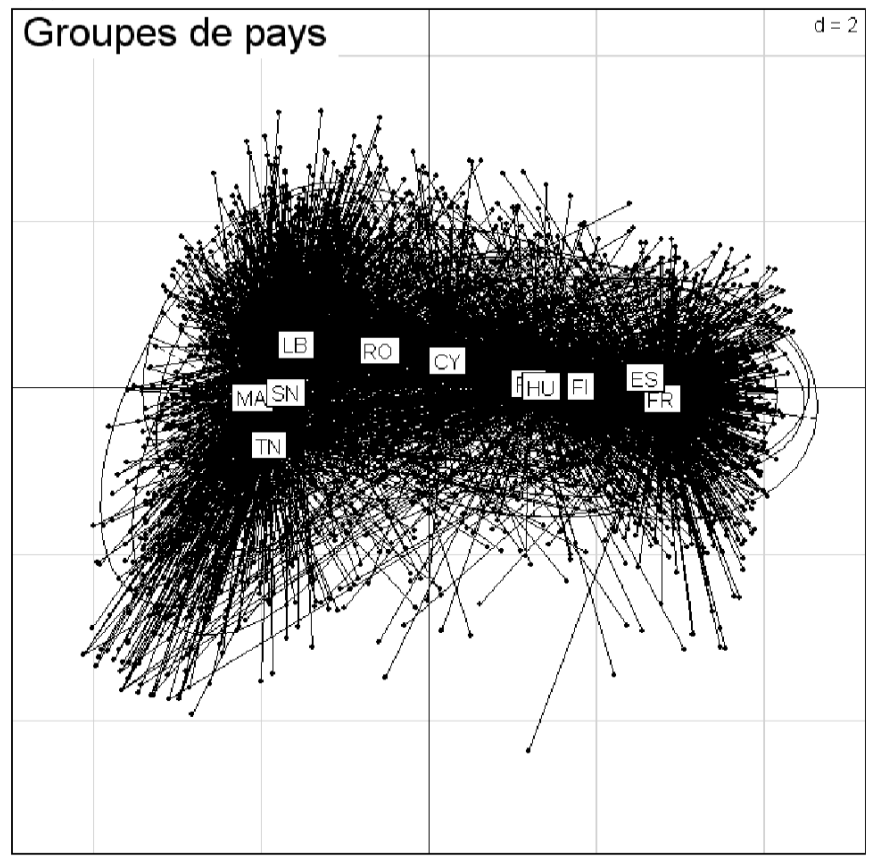

Fig. 3. Représentation des individus dans le premier plan de l'analyse discriminante sur les variables d'évolution, avec le niveau de formation (a) et le pays (b) comme variables de regroupement. Chaque individu correspond à un point. Une ellipse englobe les $2 / 3$ des membres d'un groupe et un trait relie chaque individu au centroïde figuré par un rectangle avec le nom du groupe. $1=$ enseignement secondaire $; 2=$ bac +1 ou 2 années dans le supérieur ; $3=$ bac +3 ou $4 ; 4=$ bac +5 ou $6 ; 5=$ plus élevé. Les sigles des pays sont indiqués dans le tableau : seuls 12 pays sont ici pris en compte (ceux où les enseignants ont répondu aux 15 questions sur l'évolution).

Source : Quessada et al., 2007.

s'observent dans les pays musulmans, qui sont aussi les pays non européens de nos échantillons (Tunisie, Maroc, Sénégal, Liban), et à un degré moindre dans les pays européens orthodoxes et moyennement développés sur le plan économique (Roumanie et Chypre) : Fig. 3b.

D'autres analyses, réalisées plus récemment, ont été limitées aux enseignants chrétiens de tous les pays. Elles montrent que les chrétiens français, mais aussi allemands, finlandais, etc., sont beaucoup plus évolutionnistes que les chrétiens libanais ou burkinabés, ou même chypriotes ou roumains. Les clivages relatifs au créationnisme s'observent donc au sein d'une même religion.

Une autre conclusion est à souligner : le pourcentage d'enseignants évolutionnistes augmente avec leur niveau d'instruction (Fig. 3a). Comme ce lien très net pourrait ne mesurer que la différence entre les pays européens développés et les autres pays (le niveau d'instruction des enseignants étant supérieur chez les premiers), nous avons supprimé « l'effet pays » (et «l'effet religion » fortement lié à "l'effet pays ») : la corrélation entre conviction évolutionniste et meilleur niveau d'instruction demeure alors très fortement significative $(p<0,001)$; elle existe au sein de chaque pays (voir les analyses détaillées dans Quessada et al., 2007, et Munoz et al., 2007). Ce résultat est particulièrement encourageant. Il suggère qu'une meilleure formation des enseignants, dans chaque pays, reste une arme essentielle pour lutter contre ce regain du créationnisme dont nos recherches soulignent l'ampleur.

\section{Références}

Aroua, S., 2006. Dispositif didactique pour l'enseignement de l'évolution du vivant : débat en classe pour l'enseignement de la théorie de l'évolution du vivant en Tunisie. Thèse en cotutelle ENS Cachan et ISEFC Tunis, ENS, Cachan.

Arnould, J., 2007. Dieu versus Darwin : les créationnistes vont-ils triompher de la science?, Paris, Albin Michel.

Carvalho, G., Clément, P., 2007. Construction and validation of the instruments to compare teachers' conceptions and school textbooks of 19 countries: The European BioheadCitizen project, in Hedjerassi, N., Marquet, P., AREF 2007 [Congrès international d'actualité de la recherche en éducation et en formation, Strasbourg, 28-31 août 2007], 1 vol. + 1 CD-ROM, [s.l.], [s.n.].

Clément, P., 2002. Methods to analyse argumentation in (more or less) scientific texts. An example: analysis of a text promoting Creationism, in Krnel, D. (Ed.), Proceedings of the Sixth ESERA Summerschool, 25-31 August, CD-ROM, Ljubljana, Faculty of Education, University of Ljubljana. 
Clément, P., Carvalho, G., 2007. Biology, Health and Environmental Education for better Citizenship: teachers' conceptions and textbook analysis in 19 countries, XIII World Congress of Comparative Education Societies, Sarajevo, 3-7 September.

Clément, P., Bernard, S., Castéra, J., Quessada, M.-P., 2007. Des manuels scolaires pas si neutres :

http://savoirs.essonne.fr/dossiers/les-hommes/pedagogie/ article/type/0/intro/des-manuels-scolaires-pas-si-neutres/ (Banque des savoirs).

George, S., 2007. La Pensée enchaînée, Paris, Fayard.

Lecourt, D., 1998. L'Amérique entre la Bible et Darwin, Paris, PUF.

Munoz, F., Quessada, M.-P., Clément, P., 2007. Des analyses statistiques multivariées pour traiter les données issues de questionnaires : conceptions d'enseignants et futurs enseignants de douze pays sur l'évolution, in Dusseau, J.-M., et al., Actes des Cinquièmes rencontres scientifiques de l'ARDIST, CD-ROM, La Grande-Motte, IUFM Montpellier, 297-304.

Picq, P., 2007. Lucy et l'obscurantisme, Paris, Odile Jacob.

Quessada, M.-P., Clément, P., 2006. Le quaternaire dans l'enseignement secondaire en France au $\mathrm{XIX}^{\mathrm{e}}$ et $\mathrm{XX}^{\mathrm{e}}$ siècle, Quaternaire, 18, 2, 209-214.

Quessada, M.-P., Clément, P., 2007a. An epistemological approach to French curricula on human origin during the 19th \& 20th centuries, Science E Education, 16, 9-10, 991-1006 (http://www.springerlink.com/content/97v457r12556752p/ fulltext.pdf).

Quessada, M.-P., Clément, P., 2007b. Les origines de l'homme dans les manuels scolaires français de sciences aux XIX ${ }^{\mathrm{e}}$ et $X X^{\mathrm{e}}$ siècles : interactions entre connaissances, valeurs et contexte socioculturel, in Lebrun, M., Le Manuel scolaire d'ici et d'ailleurs, d'hier à demain, 1 vol. + 1 CD-ROM, Québec, Presses de l'Université du Québec.

Quessada, M.-P., Munoz, F., Clément, P., 2007. Les conceptions sur l'évolution biologique d'enseignants du primaire et du secondaire de douze pays (Afrique, Europe et Moyen Orient) varient selon leur niveau d'étude, in Hedjerassi, N., Marquet, P., AREF 2007 [Congrès international d'actualité de la recherche en éducation et en formation, Strasbourg, 28-31 août 2007], $1 \mathrm{vol}$. +1 CD-ROM, [s.l.], [s.n.].

Quessada, M.-P., Clément, P., Oerke, B., Valente, A., à paraître. Human evolution leads to an occidental male in most of the science textbooks originate from twelve countries, Science Education International.

Zineddine, D., 2003. La Théorie de l'évolution entre connaissances scientifiques et convictions religieuses : étude des conceptions d'étudiants et de professeurs d'universités françaises et tunisiennes. Mémoire de DEA en didactique, ISEFC, Tunis. 